\title{
Antigens and Antibodies Cross-Reactive to the Murine Mammary Tumor Virus in Human Breast Cyst Fluids
}

\author{
Steven S. Witkin, Nurul H. Sarkar, David W. Kinne, Charles N. Breed, \\ Robert A. GoOD, and Noorbibi K. DAY, Memorial Sloan-Kettering Cancer \\ Center, New York 10021
}

\begin{abstract}
A B S T RA C T Human breast cyst fluids were shown to contain low concentrations of $\operatorname{IgA}(15-78 \mu \mathrm{g} / \mathrm{ml})$ and IgG $(33-145 \mu \mathrm{g} / \mathrm{ml})$. The IgA:IgG ratios in individual breast cyst fluids ranged from 1:0.6 to $1: 4$. These levels are considerably higher than their ratio in serum (1:7). IgA from $33 \%$ of the 40 fluids examined, and IgG from $10 \%$ of the fluids, reacted with the murine mammary tumor virus (MuMTV). The reactivity was detected by an enzyme-linked immunosorbent assay that measures antibody binding to both the envelope glycoprotein and core protein of the virus. In a second series of experiments, IgA from $28 \%$ of 40 breast cyst fluids reacted only with MuMTV while IgA from $30 \%$ of the fluids was reactive with both MuMTV and the Rauscher murine leukemia virus. Antigen reactive with antiserum to the 28,000-dalton MuMTV core protein (p28), was also identified in a $165,000-g$ pellet fraction from breast cyst fluids. In individual fluids, the extent of IgA binding to MuMTV was positively correlated $(P \leq 0.01)$ with the binding of anti-p28 antibody to the pellet of the breast cyst fluid. Fractions with the buoyant density of retroviruses $(1.16-1.18 \mathrm{~g} / \mathrm{ml})$ or their cores $(1.21-1.25 \mathrm{~g} / \mathrm{ml})$ were isolated from breast cyst fluids. These fractions contained a DNA polymerase capable of utilizing the reverse transcriptase-specific template, $\mathrm{dG}_{12-18} \cdot$ poly $\mathrm{rC}^{\mathrm{m}}$. In addition, they reacted with antiserum to MuMTV p28 but not with antiserum to the 30,000 dalton Rauscher murine leukemia virus core protein.
\end{abstract}

\section{INTRODUCTION}

Benign mammary cystic disease is the most common breast lesion. It occurs in $\sim 10 \%$ of women (1). Its prevalence in premenopausal women has led to the supposition that cyst formation is due to ovarian hormone stimulation (1); the actual etiological and pathogenic basis, however, remains unknown. Al-

Received for publication 2 June 1980 and in revised form 8 August 1980. though not universally accepted, several studies have demonstrated that women who have had benign mammary cysts are at an increased risk for the subsequent development of mammary adenocarcinoma (2-4).

The mammary cysts are filled with fluid. Treatment usually consists of needle aspiration of the fluids; cysts rarely recur at the site of aspiration. The cyst fluid differs significantly from serum in composition and probably arises as an exudate from epithelial cells lining the cyst $(5,6)$. The major proteins in breast cyst fluids are $(a)$ a progesterone binding glycoprotein $(6,7),(b) Z_{n}$-alpha $a_{2}$-glycoprotein, and (c) an unidentified protein with $15,000 \mathrm{~mol}$ wt immunologically unrelated to plasma proteins (6). In addition, a carcinoembryonic antigenlike protein has been identified in each of the 23 cyst fluids examined by Fleischer et al. (8). Immunoglobulin and complement components have not previously been quantitated in breast cyst fluids.

Antibodies $(9,10)$ or lymphocytes (11) reactive with murine mammary tumor virus (MuMTV) ${ }^{1}$ or antigens reactive with MuMTV-specific antisera (12) have been demonstrated in breast cancer patients. To further examine possible relationships between benign mammary cystic disease and mammary adenocarcinoma, we have analyzed breast cyst fluids for evidence of components related to MuMTV.

\section{METHODS}

Material. Aspirated breast cyst fluids were obtained by the physicians of the Breast Service at Memorial Hospital.

\footnotetext{
${ }^{1}$ Abbreviations used in this paper: BCF, breast cyst fluid; $\mathrm{dG}_{12-18} \cdot$ poly $\mathrm{rC}^{\mathrm{m}}$, a chain of $12-18$ deoxyguanylic acids complexed to a polymer of 2 '-O-methylribocytidylic acid; ELISA, enzyme-linked immunosorbent assay; gp52 and gp34, 52,000 and 34,000 dalton MuMTV surface glycoproteins; MuMTV, murine mammary tumor virus; PBS-Tween; phosphate-buffered saline containing $0.05 \%$ Tween $20 ; \mathrm{p} 28,28,000$ dalton MuMTV core proteins; p30, 30,000 dalton R-MuLV protein; R-MuLV, Rauscher murine leukemia virus.
} 
The fluids were centrifuged at $10,000 \mathrm{~g}$ for $20 \mathrm{~min}$, divided into aliquots, and stored at $-70^{\circ} \mathrm{C}$.

MuMTV (13) and rabbit or goat antiserum to the 28,000-dalton MuMTV core protein (p28), or to the Rauscher murine leukemic virus (R-MuLV) 30,000 dalton core protein (p30), were prepared as previously described (14). In pilot experiments utilizing $0.5 \mu \mathrm{g} \mathrm{MuMTV}$ or $\mathrm{R}-\mathrm{MuLV}$ protein, it was determined that a 1:180 dilution of the goat anti-MuMTV p28 serum reacted with MuMTV to give an absorbance of 0.80 and with R-MuLV to give an absorbance of 0.11 , as determined by our enzyme-linked immunosorbent assay (see below). Goat anti-R-MuLV p30 serum yielded absorbances of 0.124 and 1.346 with MuMTV and R-MuLV, respectively. The rabbit antisera exhibited similar titers.

Immunochemical analyses. The concentration of IgG, IgA, Clq, and C3 in breast cyst fluids were determined by the method of Mancini et al. (15).

Enzyme-linked immunosorbent assay (ELISA). Details of our ELISA assay for the determination of antibodies reactive with MuMTV fixed to wells of a microtiter plate have been published (10). Immunoglobulin binding was quantitated with alkaline phosphatase-conjugated swine anti-human IgG or IgA (heavy chain-specific, Orion Diagnostics, Helsinki, Finland), or alkaline phosphataseconjugated goat anti-rabbit IgG, or rabbit anti-goat IgG (Microbiological Associates, Walkersville, Md.), by determining the absorbance of the solution at $400 \mathrm{~nm}$ after the addition of $p$-nitrophenyl phosphate. Duplicate determinations differing by $>10 \%$ were reassayed. IgG binding was proportional to MuMTV concentration over a $0.1-10-\mu \mathrm{g}$ viral protein range. Antibody to MuMTV membrane components (gp 52, gp 34) and to the major internal protein (p28), all were measurable by this assay. MuMTV antibody diluted as much as 1:3,000 was readily detectable (10).

Isolation of the MuMTV-related component. Breast cyst fluid was thawed, centrifuged at $10,000 \mathrm{~g}$ for $10 \mathrm{~min}$, and the supernate centrifuged at $160,000 \mathrm{~g}$ for $60 \mathrm{~min}$ (SW 50.1 rotor) or $100,000 \mathrm{~g}$ for $90 \mathrm{~min}$ (SW 27 rotor, Beckman Instruments, Inc., Spinco Div., Palo Alto, Calif.). The resultant pellet was resuspended in phosphatebuffered saline (PBS), layered over an $18 \%$ (wt/vol) solution of metrizamide (Accurate Chemical and Scientific Corp., Hicksville, N. Y.) and again centrifuged at either $100,000 \mathrm{~g}$ or $160,000 \mathrm{~g}$. The pellet was resuspended in PBS, layered over a $5 \mathrm{ml} 15-60 \%$ (wt/wt) gradient of sucrose in PBS and centrifuged for $16 \mathrm{~h}$ at $165,000 \mathrm{~g}$. The gradient was fractionated and density determined from refractive indices. The concentration of material having the buoyant density of MuMTV $(1.18 \mathrm{~g} / \mathrm{ml})$ or MuMTV cores $(1.21-1.25 \mathrm{~g} / \mathrm{ml})$ was achieved by pooling the relevant fractions, diluting to $<10 \%$ sucrose with $\mathrm{PBS}$ and centrifuging at $165,000 \mathrm{~g}$ for $60 \mathrm{~min}$.

DNA polymerase assays. Aliquots of the sucrose gradient

TABLE I

Immunoglobulin and Complement Levels in BCF

\begin{tabular}{lcc}
\hline Component & No. positive* $^{*}$ & Concentrationt (range) \\
\hline IgG & 26 & $83.1 \mu \mathrm{g} / \mathrm{ml}(33.0-145.0)$ \\
IgA & 24 & $40.4 \mu \mathrm{g} / \mathrm{ml}(15.0-78.0)$ \\
IgM & 2 & Not done \\
Clq & 5 & $2.1 \mu \mathrm{g} / \mathrm{ml}(1.3-4.2)$ \\
C3 & 1 & Not done \\
\hline
\end{tabular}

* 33 BCF were analyzed by immunodiffusion.

† Mean concentration of the positive samples.
TABLE II

BCF IgA or IgG Binding to MuMTV

\begin{tabular}{cll}
\hline & \multicolumn{2}{c}{ Absorbance $(400 \mathrm{~nm})^{*}$} \\
\cline { 2 - 3 } Sample No. & \multicolumn{1}{c}{ IgA } & \multicolumn{1}{c}{ IgG } \\
\hline 1 & 0.026 & 0 \\
2 & 0.025 & 0 \\
3 & 0.074 & 0 \\
4 & 0 & 0.181 \\
5 & 0.080 & 0 \\
6 & 0.025 & 0 \\
7 & 0.058 & 0 \\
8 & 0.025 & 0 \\
9 & 0.009 & 0.038 \\
10 & 0.046 & 0 \\
11 & 0.052 & 0 \\
12 & 0.037 & 0 \\
13 & 0 & 0.184 \\
14 & 0.119 & 0 \\
15 & 0.075 & 0 \\
16 & 0 & 0.055 \\
17 & 0.038 & 0 \\
$18-40$ & $0-0.012$ & 0 \\
\hline
\end{tabular}

* A background absorbance of 0.100 has been subtracted from all values.

fractions were assayed for $\mathrm{dG} \cdot \mathrm{rC}^{\mathrm{m}}$-templated DNA polymerase activity using reaction conditions optimal for MuMTV reverse transcriptase (16). The reaction mixture, final volume $100 \mu$ l, contained: $0.13 \mu \mathrm{mol} \mathrm{MnCl}_{2}, 0.1 \%$ Triton X-100, 0.10 $\mu$ mol dithiothreitol, $0.02 \mu \mathrm{mol}$ ATP, $0.5 \mu \mathrm{g} \mathrm{dG}_{12-18} \cdot$ poly $\mathrm{rC}^{\mathrm{m}}$ (P-L Biochemicals, Inc., Milwaukee, Wisc.), $0.5 \mu \mathrm{Ci}\left[{ }^{3} \mathrm{H}\right]$ deoxy guanosine triphosphate (dGTP) $(10.0 \mathrm{Ci} / \mathrm{mmol}$, New England Nuclear, Boston, Mass.), and $0.1 \mu \mathrm{mol}$ Tris- $\mathrm{HCl}, \mathrm{pH}$ 7.5. ATP was added to specifically inhibit any terminal deoxynucleotidyl transferase that may be present (17). Incubation was at $37^{\circ} \mathrm{C}$ for $60 \mathrm{~min}$. Quantitation of the acidinsoluble product formed was as described (9).

\section{RESULTS}

Immunoglobulins and complement in breast cyst fluid. Fluids from 33 patients were analyzed by immunodiffusion for IgG, IgA, IgM, Clq, and C3. The results are presented in Table $I$. Low concentrations of IgA $(15-78 \mu \mathrm{g} / \mathrm{ml})$ and IgG $(33-145 \mu \mathrm{g} / \mathrm{ml})$ were found in most of the samples. The IgA:IgG ratios in individual breast cyst fluids ranged from 1:0.6 to 1:4; the IgA:IgG ratio in human sera is $\sim 1: 7$. In contrast, only two samples had detectable levels of IgM; one contained C3, and five had Clq. The absence of some serum components and an increased IgA:IgG ratio in breast cyst fluid suggests that IgA probably is produced locally in the vicinity of the cyst.

IgA and IgG binding to MuMTV or R-MuLV. We next sought to determine whether breast cyst fluid immunoglobulins would react with MuMTV. MuMTV ( $0.5 \mu \mathrm{g}$ protein) was fixed to individual wells of a microtiter plate, incubated with 1:5 dilutions of 40 breast 
TABLE III

Reactivity of BCF IgA with MuMTV or R-MuLV

\begin{tabular}{ccc}
\hline & \multicolumn{2}{c}{ Absorbance $(400 \mathrm{~nm})^{*}$} \\
\cline { 2 - 3 } Sample No. & MuMTV & R-MuLV \\
\hline 1 & 0.365 & 0 \\
2 & 0.023 & 0 \\
3 & 0.188 & 0 \\
4 & 0.067 & 0 \\
5 & 0.947 & 0 \\
6 & 0.367 & 0 \\
7 & 0.087 & 0 \\
8 & 0.102 & 0 \\
9 & 0.553 & 0 \\
10 & 0.195 & 0 \\
11 & 0.356 & 0.004 \\
12 & 0.027 & 0.492 \\
13 & 0.095 & 0.091 \\
14 & 0.230 & 0.213 \\
15 & 0.587 & 0.728 \\
16 & 0.144 & 0.085 \\
17 & 0.051 & 0.017 \\
18 & 0.165 & 0.302 \\
19 & 0.068 & 0.085 \\
20 & 0.262 & 0.245 \\
21 & 0.498 & 0.100 \\
22 & 0.216 & 0.338 \\
23 & 0.113 & 0.327 \\
$24-40$ & 0 & 0 \\
\hline
\end{tabular}

* A background absorbance of 0.100 has been subtracted.

cyst fluids; and IgA or IgG binding to the virus was quantitated by the ELISA assay with alkaline phosphatase-conjugated antisera. The results are shown in Table II. Human sera diluted to reflect breast cyst fluid IgG or IgA concentrations yielded absorbances of up to 0.10 in our assay. This value $(0.10)$ was therefore chosen as the boundary between specific and nonspecific antibody reactivity. IgA from $33 \%$ of the fluids (13/40) yielded an absorbance $\geq 0.025$ over background. IgG from $10 \%$ of the fluids (4/40) also exhibited binding to MuMTV. No fluid was positive for both IgA and IgG binding. The extent of IgA binding to MuMTV was not related to the IgA concentration in the cyst fluid, thereby making it improbable that we were merely measuring nonspecific binding.

The specificity of IgA binding to MuMTV was next examined. MuMTV or R-MuLV were fixed to microtiter plate wells, incubated with 1:5 dilutions of 40 breast cyst fluids and IgA binding to both viruses was quantitated. The results are shown in Table III. IgA from $28 \%$ of the fluids (11/40) reacted with MuMTV but not with R-MuLV, 30\% (12/40) reacted with both viruses and $42 \%(17 / 40)$ were unreactive with either virus; no fluid reacted solely with $R$ -
MuLV. The final observed absorbances in this experiment were higher than those in Table I due to the use of a new preparation of alkaline phosphataseconjugated antisera and its utilization at a lower dilution. Titration curves of IgA binding to MuMTV are shown in Fig. 1.

Anti-MuMTV p28 serum reactivity with breast cyst fluids. Individual breast cyst fluids from 17 women were centrifuged at 10,000 and $165,000 \mathrm{~g}$. The high speed pellet fraction was resuspended in PBS, layered over $18 \%$ metrizamide, and recentrifuged. The final pellets were each suspended in carbonate buffer, $\mathrm{pH} \mathrm{9.8,} \mathrm{and} \mathrm{distributed} \mathrm{to} \mathrm{four} \mathrm{wells}$ of a microtiter plate. Following incubation at $4^{\circ} \mathrm{C}$ for $18 \mathrm{~h}$, the wells were washed with PBS-0.05 Tween 20 and assayed by our ELISA assay for reactivity with serum from either an unimmunized rabbit or from a rabbit immunized with MuMTV p28. If present, retroviruses or their cores would be found in the metrizamide pellet fraction.

The results of these experiments are shown in Fig. 2. The extent of anti-p28 serum binding to the pellet fraction minus the binding of normal serum to the fractions (0.05-0.17 absorbance) is plotted for each individual fluid. Reactivity of p28 antiserum with the fixed fractions was evident in $41 \%$ (7/17) of the samples (absorbance 0.1). Furthermore, the extent of binding was correlated $(P \leq 0.01)$ with the binding capacity of IgA from a particular fluid to MuMTV (Fig. 3).

Characterization of the breast cyst fluid MuMTVlike component. To determine if further similarities existed between MuMTV and breast cyst fluid components, fluids were fractionated according to procedures utilized to purify retroviruses and their

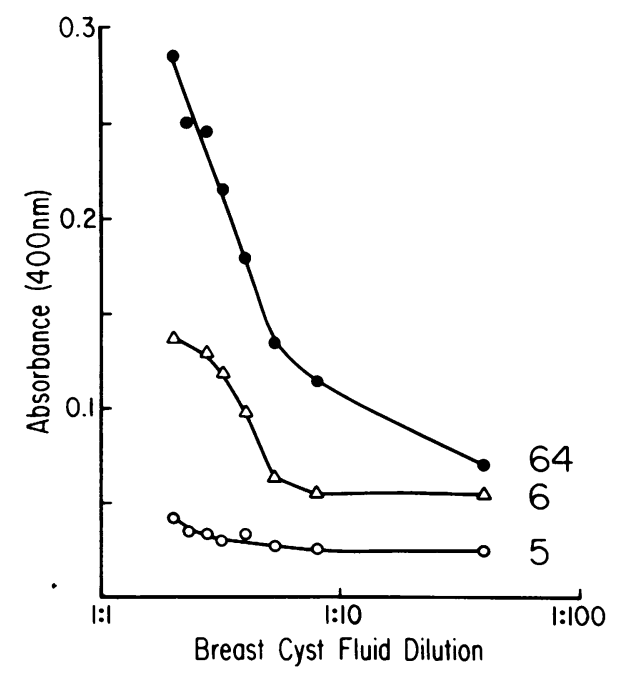

FIgURE 1 Titration of IgA reactive with MuMTV in BCF. Three BCF were diluted in PBS-Tween and assayed by the ELISA assay for IgA binding to MuMTV. 
cores from biological fluids. Intact retroviruses have a buoyant density of $1.16-1.18 \mathrm{~g} / \mathrm{ml}$, while the viral cores (nucleoids) band at $1.21-1.25 \mathrm{~g} / \mathrm{ml}$. Within the cores are RNA and RNA-instructed DNA polymerase (reverse transcriptase).

Breast cyst fluids from 11 individual women were fractionated by velocity centrifugation, pelleting through $18 \%$ metrizamide and sucrose buoyant density centrifugation, and assayed for DNA polymerase activity using the synthetic RNA templates $\mathrm{dT}_{12-18}$. poly $\mathrm{rA}$ or $\mathrm{dG}_{12-18} \cdot$ poly $\mathrm{rC}^{\mathrm{m}}$. Results for two fluids using the latter template are shown in Fig. 4. MuMTV was also analyzed in a parallel gradient. MuMTV reverse transcriptase was able to utilize $\mathrm{dG}_{12-18}$ poly $\mathrm{rC}^{\mathrm{m}}$; activity was localized mainly at the density of intact virus $(1.17 \mathrm{~g} / \mathrm{ml})$ with some activity also evident at core densities (1.21-1.25 g/ml). Both cyst fluids also exhibited $\mathrm{dG}_{12-18}$. poly $\mathrm{rC}^{\mathrm{m}}$ activity in fractions corresponding to the core density; in one case, fluid 77, activity was also present at the density of intact virus. Each of the 11 fluids exhibited DNA polymerase activity in the viral and/or core regions.

ATP was included in our assays to specifically rule out that the observed activity was due to terminal deoxynucleotidyl transferase. We had previously observed that this enzyme was present in some breast cyst fluids but that it was always localized at the top of the sucrose gradients and not in the virus or core regions (18).

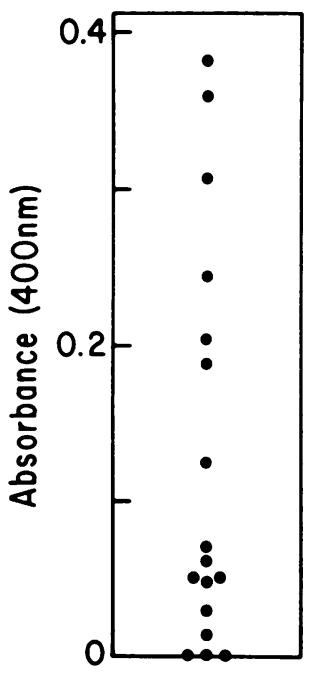

FIGURE 2 Reactivity of rabbit antiserum to MuMTV p28 with $165,000 \mathrm{~g}$ fractions from BCF. Fractions were prepared from individual BCF (Methods) and fixed in quadruplicate to wells of a microtiter plate. Two wells of each sample were incubated with sera from an unimmunized rabbit and two wells were incubated with rabbit anti-p28 serum. IgG binding was quantitated by the ELISA assay and the extent of normal serum binding (0.05-0.17 absorbance) was subtracted from the anti-p28 binding.

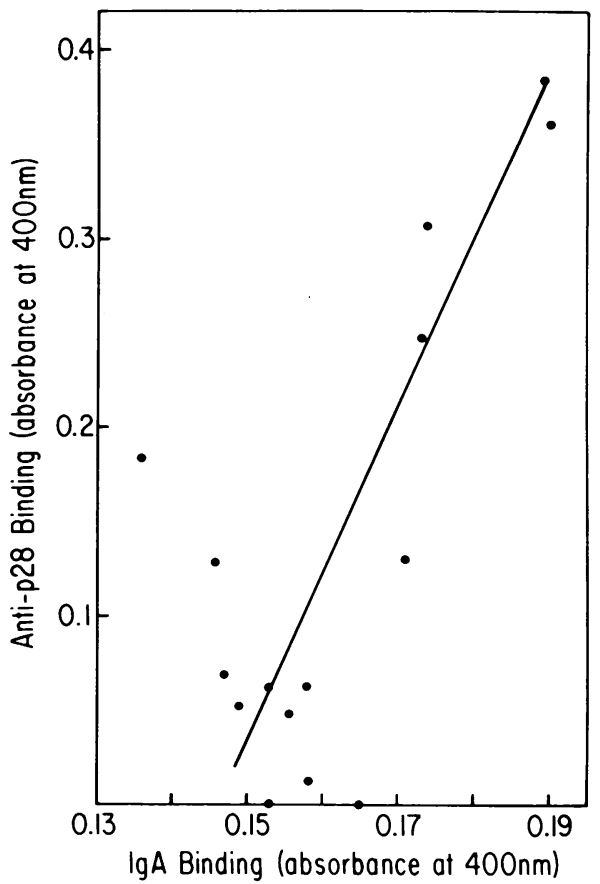

FIgURE 3 Relationship between BCF IgA binding to MuMTV and p28 anti-serum binding to BCF. IgA binding to MuMTV (Table III) vs. anti-p28 serum binding to the 165,000-g pellet fraction (Fig. 2) were plotted for individual $\mathrm{BCF}$.

Sucrose gradient fractions from six breast cyst fluids were also analyzed for reactivity with antisera to the MuMTV or R-MuLV core proteins. The fractions were concentrated by centrifugation, fixed to wells of a microtiter plate and analyzed by the ELISA assay (Table IV). Purified MuMTV was strongly reactive with antiserum to MuMTV p28 and only weakly reactive with R-MuLV p30 antiserum. The extent of reactivity of the p28 antiserum with $\mathrm{R}-\mathrm{MuLV}$ (0.221) was chosen as the boundary between specific and nonspecific reactivity. An absorbance of 0.190 resulted from the interaction of p30 antiserum with MuMTV; interaction of $\mathrm{p} 30$ antiserum with R-MuLV gave an absorbance of 0.880 . Fractions with the density of viral cores were significantly more reactive with MuMTV p28 antiserum than with R-MuLV antiserum in four of five samples; two of five fractions with the density of the virus also exhibited preferential reactivity to the MuMTV antiserum.

\section{DISCUSSION}

Low concentrations of IgA and IgG were identified in most breast cyst fluids. The absence of other serum components from breast cyst fluids plus a higher IgA:IgG ratio than that found in serum suggested 


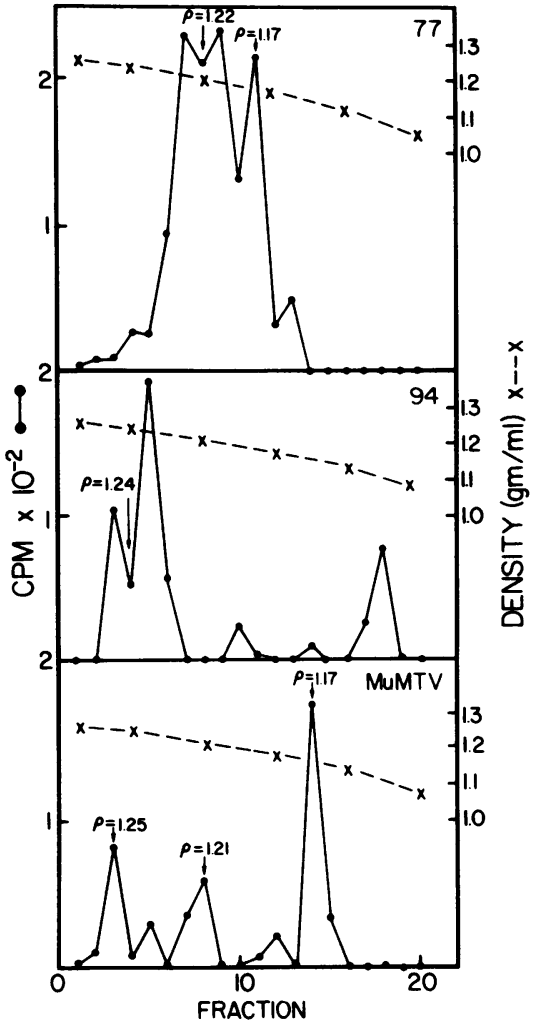

Figure 4 Buoyant density centrifugation and reverse transcriptase analysis of BCF $165,000-g$ pellet fraction. MuMTV, or the $165,000-\mathrm{g}$ pellet fraction from cyst fluids, were centrifuged in 15-60\% sucrose gradients for $16 \mathrm{~h}$, fractionated, density determined from refractive indices, and aliquots assayed for reverse transcriptase activity using $\left[{ }^{3} \mathrm{H}\right] \mathrm{dGTP}$ and $\mathrm{dG}_{12-18} \cdot$ poly $\mathrm{rC}^{\mathrm{m}}$.

that IgA was probably produced by cells in the vicinity of the cyst.

Binding of these immunoglobulins to MuMTV was then examined. IgA in breast cyst fluids was more reactive with MuMTV than was IgG. Specificity of the IgA for MuMTV was determined by comparing binding to MuMTV and to R-MuLV. Of 58\% (23/40) of breast cyst fluids that contained IgA reactive with MuMTV, half (12/23) were also reactive with R-MuLV while the remainder (11/23) were specific for MuMTV (Table III). Thus, $28 \%(11 / 40)$ of the breast cyst fluid (BCF) had IgA reactive only with MuMTV. These results indicate that there are at least two classes of BCF IgA, one reactive with both murine type B and type $C$ viruses, and a second specific for type $B$ MuMTV. Experimentation is now in progress to further define the IgA specificity for MuMTV. Initial results indicate that the reactivity is not removed by incubation of the BCF with fetal calf serum, sheep erythrocytes, or carbohydrates present in cell membranes.

If the IgA that reacted with MuMTV was produced locally, then an antigen cross-reactive with MuMTV might also be expected to be present in the cyst fluid. The antigen reacting with antiserum to the MuMTV core protein, p28, was in fact located in a $165,000-g$ pellet fraction from $41 \%$ of BCF. The extent of p28 antiserum binding to the cyst fluid pellet fraction was proportional to the reactivity of IgA in a particular fluid for MuMTV. This is consistent with a mechanism whereby IgA antibody is produced locally in response to the presence of an MuMTV-related antigen. Additional similarities between MuMTV and a component of BCF were that both were present in the 10,000-g supernate, 165,000-g pellet fractions, pelleted by centrifugation through $18 \%$ metrizamide and contained a DNA polymerase able to utilize $\mathrm{dG}_{12-18} \cdot$ poly $\mathrm{rC}^{\mathrm{m}}$ in fractions possessing buoyant densities of 1.18 to $1.25 \mathrm{~g} / \mathrm{ml}$. All available data suggest that $\mathrm{dG}_{12-18} \cdot$ poly $\mathrm{rC}^{\mathrm{m}}$ is transcribed only by reverse transcriptase (16). Cyst fluid-derived components were also shown to preferentially react with antiserum to MuMTV p28 over antiserum to $R$ MuLV p30.

It might be useful to estimate the concentration of

TABLE IV

Reactivity of BCF Sucrose Gradient Fractions with Antiserum to MuMTV p28 or R-MuLV p30

\begin{tabular}{cccc}
\hline & & \multicolumn{2}{c}{ Absorbance $(400 \mathrm{~nm})^{*}$} \\
\cline { 3 - 4 } Sample & Density & anti-p28 & anti-p30 \\
\hline & $g / m$ & & \\
BCF 29 & 1.23 & 0.441 & 0.193 \\
BCF 29 & 1.18 & 0.148 & 0.152 \\
BCF 65 & 1.23 & 0.325 & 0.181 \\
BCF 65 & 1.18 & 0.129 & 0.150 \\
BCF 68 & 1.23 & 0.171 & 0.181 \\
BCF 68 & 1.18 & 0.349 & 0.116 \\
BCF 70 & 1.23 & 0.419 & 0.242 \\
BCF 70 & 1.18 & 0.110 & 0.126 \\
BCF 80 & 1.19 & 0.439 & 0.062 \\
BCF 81 & 1.23 & 0.277 & 0.130 \\
MuMTV & 1.19 & 0.912 & 0.221 \\
\hline
\end{tabular}

$165,000-g$ pellet fractions from $1.5-3.5 \mathrm{ml} \mathrm{BCF}$ were centrifuged to equilibrium in $15-60 \%$ sucrose gradients. Fractions with viral or viral core densities were pooled, concentrated by centrifugation, fixed to wells of a microtiter plate, and assayed by the ELISA method for reactivity with goat antisera to the MuMTV or R-MuLV core proteins. Antisera titers are given in Methods.

* An absorbance $>0.221$, the reactivity of anti-p30 serum with MuMTV, is considered positive. 
MuMTV-equivalents in BCF. From the data in Table IV, $0.5 \mu \mathrm{g}$ of bound MuMTV yielded an absorbance of 0.912 in a reaction with p28 antiserum. If we assume that the absorbance varies proportionally with viral concentration, as is in fact the case (10), it can be calculated that the six fluids reactive with p28 antiserum contained 0.18-0.24 $\mu$ g MuMTV equivalent per $3 \mathrm{ml}$ fluid.

Low concentrations of particles with the morphology (19) and biochemical properties (20) or retroviruses have also been observed in some samples of human milk. They may be identical to the BCF components, since both are probably derived from mammary epithelia. Schlom et al. (20) have determined, based on nucleic acid hybridizations, that there are up to $4 \times 10^{7}$ viruslike components $/ \mathrm{ml}$ of human milk. If we estimate that a single retrovirus component is $7 \times 10^{-10}$ $\mu \mathrm{g}$, then we can calculate that there may be up to $0.03 \mu \mathrm{g}$ virus $/ \mathrm{mg}$ milk. This is comparable to the $0.06-$ $0.08 \mu \mathrm{g}$ components $/ \mathrm{ml}$ we estimated to be present in BCF (see above). Whether these components originate from exogenous infection or represent expression of endogenous genetic information, is still unclear.

Mesa-Tejada et al. (12) reported that paraffin sections of 119 benign breast lesions were unreactive with antibody to MuMTV. This difference from our results may possibly be due to the greater accumulation of MuMTV-related antigen in the cyst fluids than in the surrounding tissue. Furthermore, components related to the MuMTV core protein, $\mathrm{p} 28$, have, to our knowledge, not been detected in human breast cancers. A p28-like protein has been identified, however, in human milk particles (21).

Because only a fraction of the $35 \%$ of women with detectable MuMTV-related components in their cyst fluids would be expected to develop mammary adenocarcinoma, it appears that the presence of these components is not sufficient by itself to induce malignancy. Perhaps additional genetic and/or environmental factors are needed to complete the transformation process. Evaluation of potential deleterious or beneficial consequences of the appearance of these components in the mammary gland awaits further characterization of their immunological, biochemical, and biological properties.

\section{ACKNOWLEDGMENTS}

We are indebted to the members of the Breast Service at Memorial Hospital for their interest and cooperation. Ms. Ann Marie Fegorello provided expert technical assistance. We also thank Ms. Patsey Yeo-Ramaker, R.N. for sample and data coordination.

These studies were supported by grants from the Memorial Sloan-Kettering Cancer Center Special Projects Committee,
CA 08748, AI 11843, NS 11457, Sherman Fairchild Foundation, and the Zelda Radow Weintraub Foundation.

\section{REFERENCES}

1. Haagensen, C. D. 1971. Cystic disease of the breast. In Diseases of the Breast. C. D. Haagensen, editor. W. B. Saunders, Philadelphia. 156-176.

2. Lewison, E. F., and L. G. Lyons. 1953. Relationship between benign breast disease and cancer. Arch. Surg. 66: $94-114$.

3. Veronesi, V., and G. Pizzocaro. 1968. Breast cancer in women subsequent to cystic disease of the breast. Surg. Gynecol. Obstet. 126: 529-532.

4. Block, M. M., T. H. C. Barclay, S. J. Cutter, B. F. Hankey, and A. J. Asire. 1972. Association of atypical characteristics of benign breast lesions with subsequent risk of breast cancer. Cancer (Phila.). 29: 338-343.

5. Fleisher, M., G. F. Robbins, C. N. Breed, Jr., A. A. Fracchia, J. A. Urban, and M. K. Schwartz. 1973. Biochemistry of breast cyst fluid. Memorial Sloan-Kettering Cancer Center Clinical Bulletin. 3: 94-97.

6. Haagensen, D. E., Jr., G. Mazoujian, W. G. Dilley, C. E. Pederson, S. J. Kister, and S. A. Wells, Jr. 1979. Breast gross cystic disease fluid analysis. I. Isolation and radioimmunoassay for a major component protein. J. Natl. Cancer Inst. 62: 239-247.

7. Pearlman, W. H., J. L. Gueriguian, and M. E. Sawyer. 1973. A specific progesterone-binding component of human breast cyst fluid. J. Biol. Chem. 248: 5736-5741.

8. Fleisher, M., H. F. Oettgen, C. N. Breed, G. F. Robbins, C. M. Pinsky and M. K. Schwartz. 1974. CEA-like material in fluid from benign cysts of the breast. Clin. Chem. 20: 41-42.

9. Witkin, S. S., R. A. Egeli, N. H. Sarkar, R. A. Good, and N. K. Day. 1979. Virolysis of mouse mammary tumor virus by sera from breast cancer patients. Proc. Natl. Acad. Sci. U. S. A. 76: 2984-2987.

10. Witkin, S. S., N. H. Sarkar, R. A. Good, and N. K. Day. 1980. An enzyme-linked immunoassay for the detection of antibodies to the mouse mammary tumor virus: application to human breast cancer. J. Immunol. Methods. 32: $85-91$.

11. Black, M. M., R. E. Zachrau, B. Shore, D. H. Moore, and H. P. Leis, Jr. 1975. Prognostically favorable immunogens of human breast cancer tissue: antigenic similarity to murine mammary tumor virus. Cancer (Phila.). 35: $121-128$.

12. Mesa-Tejada, R., I. Keydar, M. Ramanarayanan, T. Ohno, C. Fenoglio, and S. Spiegelman. 1978. Detection in human breast carcinomas of an antigen immunologically related to a group-specific antigen of mouse mammary tumor virus. Proc. Natl. Acad. Sci. U. S. A. 75: 1529-1533.

13. Sarkar, N. H., A. A. Pomenti, and A. S. Dion. 1977. Replication of mouse mammary tumor virus in tissue culture. I. Establishment of a mouse mammary tumor cell line, virus characterization and quantitation of virus production. Virology. 77: 12-30.

14. Marcus, S. L., S. W. Smith, J. Racevskis, and N. Sarkar. 1978. The relative hydrophobicity of oncornaviral structural proteins. Virology. 86: 398-412.

15. Mancini, G., A. V. Carbonara, and J. F. Heremans. 1965. Immunochemical quantitation of antigen by single radial immunodiffusion. Immunochemistry. 2: 235-254. 
16. Marcus, S. L., N. H. Sarkar, and M. J. Modak. 1976. Purification and properties of murine mammary tumor virus DNA polymerase. Virology. 71: 242-254.

17. Bhalla, R. B., M. K. Schwartz, and M. J. Modak. 1977. Selective inhibition of terminal deoxynucleotidyl transferase (TdT) by adenosine ribonucleoside triphosphate (ATP) and its application in the detection of TdT in human leukemia. Biochem. Biophys. Res. Commun. 76: 1056-1061.

18. Witkin, S. S., E. Borenfreund, A. Bendich, M. Fleisher, and M. K. Schwartz. 1978. Extracellular DNA polymerase from breast cyst fluids and derived cell cultures. Proc. Am. Assoc. Cancer Res. 19: 175. (Abstr.)
19. Moore, D. H., J. Charney, B. Kramarsky, E. Y. Lasfargues, N. H. Sarkar, M. J. Brennan, J. H. Burrows, S. M. Sirsat, J. C. Paymaster, and A. B. Vaidya. 1971. Search for a human breast cancer virus. Nature (Lond.). 229: 611-615.

20. Schlom, J., D. Colcher, S. Spiegelman, S. Gillespie, and D. Gillespie. 1973. Quantitation of RNA tumor viruses and viruslike particles in human milk by hybridization to polyadenylic acid sequences. Science (Wash. D. C.). 179: 696-698.

21. Furmanski, P., C. P. Loeckner, C. Longley, L. J. Larson, and M. A. Rich. 1976. Identification and isolation of the major core protein from the oncornavirus-like particle in human milk. Cancer Res. 36: 4001-4007. 